\title{
Midazolam versus neostigmine as adjuvants to bupivacaine in ultrasound guided supraclavicular brachial plexus block: relationship to 8-epi-prostaglandin F2 alpha and inflammatory biomarkers \\ Sayed S. ${ }^{1}$, Idriss N. ${ }^{2}$, Ahmed R. ${ }^{1}$ \\ ${ }^{1}$ Assuit University, Dept of Anesthesiology \& Intensive Care, Assuit, Egypt, \\ ${ }^{2}$ Assuit University, Medical Biochemistry and Molecular Biology, Assuit, Egypt
}

\section{Background and Goal of Study}

Brachial plexus block is a practical surrogate to general anaesthesia.

Postoperative analgesia is an added benefit. Comparing the efficacy of midazolam and neostigmine addition to bupivacaine in supraclavicular brachial plexus block as regard the onset and duration of sensory and motor block and duration of analgesia.

\section{Materials and Methods}

A prospective, randomized, study was done at Assuit university Hospital.

Ninety adult patients ASA I,II and III ,aged between 18-65 years and scheduled for various upper limb surgeries except shoulder surgeries under ultrasound guided supraclavicular blockade.

Patients were divided into three groups 30 each:

$>$ group $1(\mathrm{n}=30)$, $30 \mathrm{ml}$ bupivacaine $0.5 \%$

$>$ group $2(\mathrm{n}=30), 28 \mathrm{ml}$ bupivacaine $0.5 \%+2 \mathrm{ml}$ midazolam $(50 \mathrm{mic} / \mathrm{kg}$ )

> group 3 ( $\mathrm{n}=30$ ), $28 \mathrm{ml}$ bupivacaine $+2 \mathrm{ml}$ neostigmine (200mic).

Biochemical indices such as

$>$ serum human 8-epi-prostaglandin F2alph (8-epi PGF2),

$>$ serum cortisol,

$>$ plasma Interleukin 6 \&8 were measured using ELISA.

As well as Plasma vitamin C concentrations were determined by HPLC.

Hemodynamic variables, oxygen saturation as well as pain scores, sedation scores, motor and sensory block, onset times, block durations were measured.

\section{Results}

No significant difference in the demographic data and surgical characteristics in the three groups

The onset and duration of sensory and motor block was significantly faster and longer in group 2 compared to other groups $(p<0.001)$.

Pain score and requirement of salvage analgesia were significantly lower in group 1 compared to the other groups.

Serum levels of 8-epi PGF2, cortisol,Interleukin 6 \&8 and plasma vitamin C concentrations showed no significant difference in three groups preoperatively.

Postoperatively, there were significant lower levels of serum 8-epi PGF2, cortisol interleukin-8 and plasma Vit $C$ in group 2 compared to group $1(p<0.001)$ and group $3(p<0.01)$ respectively. However Interleukin 6 showed no significant difference.

\section{Conclusions}

Addition of midazolam or neostigmine to bupivacaine for supraclavicular brachial plexus block prolonged sensory blockade and post-operative analgesia without rising the danger of unsympathetic effects, delayed time to first analgesic use and decreases total analgesic requirements without side-effects. In addition the use of ultrasound guidance brachial plexus block presented a significant suppression of several biochemical indices. 